$\mathrm{J}$ o u r n a l of

Mathematics

and Applications

JMA No 40, pp 37-58 (2017)

\title{
Existence and Controllability Results for Sobolev-type Fractional Impulsive Stochastic Differential Equations with Infinite Delay
}

\author{
Ahmed Boudaoui and Abdeldjalil Slama
}

\begin{abstract}
In this paper, we prove the existence of mild solutions for Sobolev-type fractional impulsive stochastic differential equations with infinite delay in Hilbert spaces. In addition, the controllability of the system with nonlocal conditions and infinite delay is studied. An example is provided to illustrate the obtained theory.
\end{abstract}

AMS Subject Classification: 65C30, 93B05, 34K40, 34K45.

Keywords and Phrases: Fractional impulsive stochastic differential equations; Fixed point principle; Mild solution; Controllability.

\section{Introduction}

Stochastic differential equations is an important emerging field and has attracted great interest from both theoretical and applied disciplines, which has been successfully applied to problems in physics, biology, chemistry, mechanics and so on (see $[6,7,9$, $14,21]$ ) and the references therein). In the present literature, there are many papers on the existence and uniqueness of solutions to stochastic differential equations (see $[2,3$, 25]). The stochastic differential equations with infinite delay have become important in recent years as mathematical models of phenomena in both physical and social sciences [26, 28]. The existence of mild solutions and (approximate) controllability for different types of fractional evolution systems have been reported by many researchers (see $[5,17,19,25,27,28,29]$ and the references therein).

The Sobolev type (fractional) equation appears in a variety of physical problems such as flow of fluid through fissured rocks, thermodynamics, propagation of long waves of small amplitude and shear in second order fluids and so on [20]. There are many interesting results on the the existence and uniqueness of mild solutions and

COPYRIGHT (c) by Publishing House of Rzeszów University of Technology P.O. Box 85, 35-959 Rzeszów, Poland 
approximate controllability for a class of Sobolev type fractional evolution equations, we refer the reader to $[1,11,15,18,20]$.

Recently, the existence of mild solutions and the approximate controllability of fractional Sobolev type evolution system in Banach spaces have been studied in many publications (see $[11,12,13,18,15]$ and the references therein).

More recently, Benchaabane and Sakthivel [4] investigated the existence and uniqueness of mild solutions for a class of nonlinear fractional Sobolev type stochastic differential equations in Hilbert spaces. A new set of sufficient condition is established with the coefficients in the equations satisfying some non-Lipschitz conditions. Revathi et al. [22] studied the local existence of mild solution for a class of stochastic functional differential equations of Sobolev-type with infinite delay. The results are extended to study the local existence results for neutral stochastic differential equations of Sobolev-type.

For our knowledge, there is no work reported on Sobolev-type fractional impulsive stochastic differential equation with infinite delay. Motivated by the above works, the purpose of this paper is to prove the existence and uniqueness of mild solutions and the controllability for the Sobolev-type fractional impulsive stochastic differential equation with infinite delay. Our approach is based on the fixed point theorem. The rest of this paper is organized as follows. In Section 2, we will provide some basic definitions, lemmas and basic properties of fractional calculus. The concept of mild solutions, a set of sufficient conditions for the existence and uniqueness of mild solutions for the considered equations is obtained in Section 3. In Section 4, provide a sufficient condition for the controllability for a class of fractional evolution equations of Sobolev-type impulsive stochastic fractional equations with nonlocal conditions and infinite delay.

\section{Preliminaries and basic properties}

Let $\mathcal{H}, \mathcal{K}$ be two separable Hilbert spaces and $L(\mathcal{K}, \mathcal{H})$ be the space of bounded linear operators from $\mathcal{K}$ into $\mathcal{H}$. For convenience, we will use the same notation $\|$. $\|$ to denote the norms in $\mathcal{H}, \mathcal{K}$ and $L(\mathcal{K}, \mathcal{H})$, and use $(.,$.$) to denote the inner product$ of $\mathcal{H}$ and $\mathcal{K}$ without any confusion. Let $\left(\Omega, \mathcal{F},\left\{\mathcal{F}_{t}\right\}_{t \geq 0}, \mathbb{P}\right)$ be a complete filtered probability space satisfying that $\mathcal{F}_{0}$ contains all $\mathbb{P}$-null sets of $\mathcal{F} . \omega=\left(\omega_{t}\right)_{t \geq 0}$ be a $Q$-Wiener process defined on $\left(\Omega, \mathcal{F},\left\{\mathcal{F}_{t}\right\}_{t>0}, \mathbb{P}\right)$ with the covariance operator $Q$ such that $\operatorname{Tr} Q<\infty$. We assume that there exists a complete orthonormal system $\left\{e_{k}\right\}_{k>1}$ in $\mathcal{K}$, a bounded sequence of nonnegative real numbers $\lambda_{k}$ such that $Q e_{k}=\lambda_{k} e_{k}$, $k=1,2, \cdots$, and a sequence of independent Brownian motions $\left\{\beta_{k}\right\}_{k \geq 1}$ such that

$$
(\omega(t), e)_{\mathcal{K}}=\sum_{k=1}^{\infty} \sqrt{\lambda_{k}}\left(e_{k}, e\right)_{\mathcal{K}} \beta_{k}(t), \quad e \in \mathcal{K}, \quad t \geq 0 .
$$

Let $\mathcal{L}_{2}^{0}=\mathcal{L}_{2}\left(\mathbb{Q}^{\frac{1}{2}} \mathcal{K}, \mathcal{H}\right)$ be the space of all HilbertSchmidt operators from $\mathbb{Q}^{\frac{1}{2}} \mathcal{K}$ to $\mathcal{H}$ with the inner product $\left\langle\varphi, \psi>_{\mathscr{L}_{2}^{0}}=\operatorname{Tr}\left[\varphi Q \psi^{*}\right]\right.$. 
In this paper, we consider the following Sobolev-type fractional impulsive stochastic differential equations with infinite delay:

$$
\left\{\begin{array}{l}
\left.D_{t}^{\alpha} L x(t)=A x(t)+f\left(t, x_{t}\right)\right)+\sigma\left(t, x_{t}\right) \frac{d \omega(t)}{d t}, \quad t \in J=[0, T], T>0, t \neq t_{k} \\
\Delta x\left(t_{k}\right)=I_{k}\left(x\left(t_{k}^{-}\right)\right), \quad k=1, \cdots, m \\
x(t)=\phi, \quad \phi \in B_{h}
\end{array}\right.
$$

where $D_{t}^{\alpha}$ is the Caputo fractional derivative of order $\alpha, \frac{1}{2}<\alpha<1, x($.$) takes the$ value in a separable Hilbert space $\mathcal{H}$. We assume that the operators $L$ and $A$ are defined as: $A: D(A) \in \mathcal{H} \rightarrow \mathcal{H}$ and $L: D(L) \in \mathcal{H} \rightarrow \mathcal{H}$ generates a strongly continuous semigroup $S(t)_{t \geq 0}$. Here $0=t_{0} \leq t_{1} \leq \cdots \leq t_{m} \leq t_{m+1}=T, \Delta x\left(t_{k}\right)=$ $I_{k}\left(x\left(t_{k}^{-}\right)\right)=x\left(t_{k}^{+}\right)-x\left(t_{k}^{-}\right), x\left(t_{k}^{+}\right)=\lim _{h \rightarrow 0} x\left(t_{k}+h\right)$ and $x\left(t_{k}^{-}\right)=\lim _{h \rightarrow 0} x\left(t_{k}-h\right)$ represent the right and left limits of $x(t)$ at $t=t_{k}$ respectively. The initial data $\phi=\{\phi(t) ; t \in(-\infty, 0]\}$ is an $\mathcal{F}_{0}$-measurable, $\mathcal{B}_{h}$-valued random variable independent of $\omega$ with finite second moments. Further $f: J \times \mathcal{B}_{h} \rightarrow \mathcal{H}$ and $\sigma: J \times \mathcal{B}_{h} \rightarrow \mathcal{L}_{2}^{0}(\mathcal{K}, \mathcal{H})$ are appropriate mappings will be specified later.

We introduce the following assumptions on the operators $L$ and $A$.

L1 $L$ and $A$ are closed linear operators,

L2 $D(L) \subset D(A)$ and $L$ is bijective,

L3 $L^{-1}: \mathcal{H} \rightarrow D(L)$ is compact.

Remark 2.1. From (L3), we deduce that $L^{-1}$ is a bounded operators, for short, we denote by $C_{1}=\left\|L^{-1}\right\|$ and $C_{2}=\|L\|$. Note $(L 3)$ also implies that $L$ is closed since the fact: $L^{-1}$ is closed and injective, then its inverse is also closed. It comes from $(L 1)-(L 3)$ and the closed graph theorem, we obtain the boundedness of the linear operator $A L^{-1}: \mathcal{H} \rightarrow \mathcal{H}$. Consequently, $-A L^{-1}$ generates a strongly continuous semigroup $\{S(t)\}_{t \geq 0}$ in $\mathcal{H}$. We suppose that $M:=\max _{t \in[0, T]}\|S(t)\|$.

Now, we present the abstract space phase $\mathcal{B}_{h}$. Assume that $h:(-\infty, 0] \rightarrow(0,+\infty)$ with $l=\int_{-\infty}^{0} h(t) d t<\infty$ a continuous function. We define the abstract phase space $\mathcal{B}_{h}$ by

$$
\mathcal{B}_{h}:=\left\{\phi:(-\infty, 0] \times \Omega \rightarrow \mathcal{H}, \text { for any } a>0,\left(E \mid \phi\left(\left.\theta\right|^{2}\right)^{\frac{1}{2}}\right.\right.
$$

is bounded and measurable function on $[-a, 0]$ and

$$
\int_{-\infty}^{0} h(s) \sup _{s \leq \theta \leq 0}\left(E \| \phi\left(\theta \|^{2}\right)^{\frac{1}{2}}<+\infty\right\} .
$$

If $\mathcal{B}_{h}$ is endowed with the norm

$$
\|\phi\|_{\mathcal{B}_{h}}:=\int_{-\infty}^{0} h(s) \sup _{s \leq \theta \leq 0}\left(E\|\phi(\theta)\|^{2}\right)^{\frac{1}{2}}, \phi \in \mathcal{B}_{h},
$$


then $\left(\mathcal{B}_{h},\|\cdot\|_{\mathcal{B}_{h}}\right)$ is a Banach space $[23,24]$.

Now we consider the space

$$
\mathcal{B}_{T}:=\left\{x:(-\infty, T] \times \Omega \rightarrow \mathcal{H}, \quad \text { such that }\left.\quad x\right|_{J_{k}} \in C\left(J_{k}, \mathcal{H}\right)\right.
$$

and there exist $x\left(t_{k}^{+}\right), \quad$ and $\quad x\left(t_{k}^{-}\right)$with $\quad x\left(t_{k}\right)=x\left(t_{k}^{-}\right), x_{0}=\phi \in \mathcal{B}_{h}, k=1, \ldots, m$

$$
\text { and } \left.\sup _{0 \leq s \leq T}\left(E\|x(s)\|^{2}\right)<\infty\right\}
$$

where $\left.x\right|_{J_{k}}$ is the restriction of $x$ to $J_{k}=\left(t_{k}, t_{k+1}\right], k=0,1,2, \ldots, m$. We endow a seminorm $\|\cdot\|_{\mathcal{B}_{T}}$ on $\mathcal{B}_{T}$, it is defined by

$$
\|x\|_{\mathcal{B}_{T}}=\|\phi\|_{\mathcal{B}_{h}}+\sup _{0 \leq s \leq T}\left(E\|x(s)\|^{2}\right)^{\frac{1}{2}}, x \in \mathcal{B}_{T} .
$$

We recall the following lemma:

Lemma 2.2. [24] Assume that $x \in \mathcal{B}_{T}$; then for $t \in J, x_{t} \in \mathcal{B}_{h}$. Moreover

$$
\left.l\left(E\|x(t)\|^{2}\right)^{\frac{1}{2}} \leq l \sup _{s \in[0, t]} E\|x(s)\|^{2}\right)^{\frac{1}{2}}+\left\|x_{0}\right\|_{\mathcal{B}_{h}},
$$

where $l=\int_{-\infty}^{0} h(s) d s<\infty$.

Definition 2.3. [8] The Caputo derivative of order $\alpha$ for a function $f:[0, \infty) \rightarrow \mathbb{R}$, which is at least $n$-times differentiable can be defined as

$$
D_{a}^{\alpha} f(t)=\frac{1}{\Gamma(n-\alpha)} \int_{a}^{t}(t-s)^{n-\alpha-1} f^{(n)}(s) d s=I_{a}^{(n-\alpha)}\left(\frac{d^{n} f}{d t^{n}}\right)(t)
$$

for $n-1 \leq \alpha<n, n \in \mathbb{N}$. If $0<\alpha \leq 1$, then

$$
D_{a}^{\alpha} f(t)=\frac{1}{\Gamma(1-\alpha)} \int_{a}^{t}(t-s)^{-\alpha}\left(\frac{d f(s)}{d s}\right) d s .
$$

Obviously, the Caputo derivative of a constant is equal to zero.

Definition 2.4. The fractional integral of order $\alpha$ with the lower limit 0 for a function $f$ is defined as

$$
I^{\alpha} f(t)=\frac{1}{\Gamma(\alpha)} \int_{0}^{t}(s-t)^{\alpha-1} f(s) d s
$$

provided the right-hand side is pointwise defined on $[0, \infty)$, where the $\Gamma$ is the gamma function.

Remark 2.5. If $f$ is an abstract function with values in $\mathcal{H}$, then integrals which appear in Definition 2.4 are taken in Bochners sense. 

by

For $x \in \mathcal{H}$, we define two families $\left\{T_{L}(t), t \geq 0\right\}$ and $\left\{S_{L}(t), t \geq 0\right\}$ of operators

$$
\begin{gathered}
T_{L}(t):=T_{\alpha}(t) L^{-1}=\int_{0}^{\infty} L^{-1} \Psi_{\alpha}(\theta) S\left(t^{\alpha} \theta\right) d \theta, \\
S_{L}(t):=S_{\alpha}(t) L^{-1}=\alpha \int_{0}^{\infty} L^{-1} \theta \Psi_{\alpha}(\theta) S\left(t^{\alpha} \theta\right) d \theta,
\end{gathered}
$$

where

$$
\left.\Psi_{\alpha}(\theta):=\frac{1}{\pi \alpha} \sum_{n=1}^{\infty}(-\theta)^{n-1} \frac{\Gamma(n \alpha+1)}{n !} \sin (n \pi \alpha), \quad \theta \in\right] 0,+\infty[
$$

is a probability density function defined on $] 0, \infty\left[\right.$, which satisfies that $\Psi_{\alpha}(\theta) \geq 0$ and $\int_{0}^{\infty} \Psi_{\alpha}(\theta) d \theta=1$.

Lemma 2.6. [30] The operators $T_{\alpha}$ and $S_{\alpha}$ have the following properties:

1. For any fixed $x \in \mathcal{H},\left\|T_{\alpha}(t) x\right\| \leq M\|x\|,\left\|S_{\alpha}(t) x\right\| \leq \frac{M}{\Gamma(\alpha)}\|x\|$.

2. $\left\{T_{\alpha}(t), t \geq 0\right\}$ and $\left\{S_{\alpha}(t), t \geq 0\right\}$ are strongly continuous.

Lemma 2.7. [13] The operators $T_{L}$ and $S_{L}$ defined by (2.5) have the following properties:

1. For any fixed $t \geq 0, T_{L}(t)$ and $S_{L}(t)$ are linear and bounded operators, and for any $x \in \mathcal{H}$

$$
\begin{aligned}
\left\|T_{L}(t) x\right\| & \leq M C_{1}\|x\|, \\
\left\|S_{L}(t) x\right\| & \leq \frac{M C_{1}}{\Gamma(\alpha)}\|x\| .
\end{aligned}
$$

2. $\left\{T_{L}(t), t \geq 0\right\}$ and $\left\{S_{L}(t), t \geq 0\right\}$ are compact.

The key tool in our approach is the following form of the Krasnoselskii's fixed point theorem [10].

Theorem 2.8. Let $B$ be a nonempty closed convex of a Banach space $(X,\|\|$.$) . Sup-$ pose that $P$ and $Q$ map $B$ into $X$ such that

(i) $P x+Q y \in B$ whenever $x, y \in B$;

(ii) $P$ is compact and continuous;

(iii) $Q$ is a contraction mapping.

Then there exists $z \in B$ such that $z=P z+Q z$. 


\section{Existence of mild solutions}

In this section, we first establish the existence of mild solutions to Sobolev-type fractional stochastic differential equations (2.1). More precisely, we will formulate and prove sufficient conditions for the existence of solutions to (2.1) with infinite delay and impulses. First, we first define the concept of mild solution to our problem.

Definition 3.1. A cádlàg $\mathcal{H}$-valued process $x$ is said to be a mild solution of $(2.1)$ if

1. $x(t)$ is $\mathcal{F}_{t}$-adapted and $\{x(t), t \in[0, T]\}$ is $\mathcal{B}_{h}$-valued,

2. for each $t \in J, x(t)$ satisfies the following integral equation:

$$
\begin{gathered}
x(t)=T_{L}(t) L \phi(0)+\int_{0}^{t}(t-s)^{\alpha-1} S_{L}(t-s) f\left(s, x_{s}\right) d s \\
+\int_{0}^{t}(t-s)^{\alpha-1} S_{L}(t-s) \sigma\left(s, x_{s}\right) d \omega(t) \\
\quad+\sum_{0<t_{k}<t} T_{L}\left(t-t_{k}\right) I_{k}\left(x\left(t_{k}^{-}\right)\right),
\end{gathered}
$$

3. $x(t)=\phi(t)$ on $(-\infty, 0]$ satisfying $\|\phi\|_{\mathcal{B}_{h}}^{2}<\infty$.

Let us introduce the following hypotheses:

(H1) There exists $L_{f}>0$ such that

$$
E\|f(t, x)-f(t, y)\|_{\mathcal{H}}^{2} \leq L_{f}\|x-y\|_{\mathcal{B}_{h}}^{2}, \quad t \geq 0 .
$$

(H2) There exists $L_{\sigma}>0$ such that

$$
E\|\sigma(t, x)-\sigma(t, y)\|_{\mathscr{L}_{2}^{0}}^{2} \leq L_{\sigma}\|x-y\|_{\mathcal{B}_{h}}^{2}, \quad t \geq 0
$$

(H3) For all $x \in \mathcal{H}$, there exist constants $L_{k}>0, k=1, \ldots, m, \ldots$ for each

$$
\left|I_{k}(y)\right|^{2} \leq L_{k}
$$

Theorem 3.2. Assume that $f(t, 0)=\sigma(t, 0)=0, \forall t \geq 0$. Assume that hypotheses $(H 1)-(H 3)$ hold. If

$$
r \geq 3 M^{2} C_{1}^{2} \sum_{k=1}^{m} L_{k}+\frac{3 M^{2} C_{1}^{2} T^{2 \alpha} \chi}{\Gamma^{2}(\alpha)}\left[\frac{L_{f}}{\alpha^{2}}+\frac{L_{\sigma}}{T(2 \alpha-1)}\right]
$$

and

$$
\frac{2 M^{2} C_{1}^{2}}{\Gamma^{2}(\alpha)} T^{2 \alpha}\left[\frac{L_{f} l}{\alpha^{2}}+\frac{L_{\sigma} l}{T(2 \alpha-1)}\right]<1,
$$

then system (2.1) has a mild solution on $(-\infty, T]$. 
Proof. Transform the problem (2.1) into a fixed-point problem. Consider the operator $\Psi: \mathcal{B}_{T} \rightarrow \mathcal{B}_{T}$ defined by

$$
\Psi x(t)=\left\{\begin{array}{l}
\phi(t), \quad \text { if } \quad t \in(-\infty, 0], \\
T_{L}(t) L \phi(0)+\int_{0}^{t}(t-s)^{\alpha-1} S_{L}(t-s) f\left(s, x_{s}\right) d s \\
+\int_{0}^{t}(t-s)^{\alpha-1} S_{L}(t-s) \sigma\left(s, x_{s}\right) d W(t) \\
+\sum_{0<t_{k}<t} T_{L}\left(t-t_{k}\right) I_{k}\left(x\left(t_{k}^{-}\right)\right) .
\end{array}\right.
$$

For $\phi \in \mathcal{B}_{h}$, we define $\widehat{\phi}$ by

$$
\widehat{\phi}(t)=\left\{\begin{array}{cc}
\phi(t), & t \in(-\infty, 0], \\
T_{L}(t) L \phi(0), & t \in[0,+\infty[;
\end{array} \text { then } \widehat{\phi} \in \mathcal{B}_{T} .\right.
$$

Let $x(t)=y(t)+\widehat{\phi}(t),-\infty<t<T$.

It is evident that $y$ satisfies that $y_{0}=0, t \in(-\infty, 0]$ and

$$
\begin{aligned}
y(t) & =\int_{0}^{t}(t-s)^{\alpha-1} S_{L}(t-s) f\left(s, y_{s}+\widehat{\phi}_{s}\right) d s \\
& +\int_{0}^{t}(t-s)^{\alpha-1} S_{L}(t-s) \sigma\left(s, y_{s}+\widehat{\phi}_{s}\right) d \omega(t) \\
& +\sum_{0<t_{k}<t} T_{\alpha}\left(t-t_{k}\right) I_{k}\left(y\left(t_{k}^{-}\right)+\widehat{\phi}\left(t_{k}^{-}\right)\right), t \in J
\end{aligned}
$$

if and only if $x$ satisfies that $x(t)=\phi(t), t \in(-\infty, 0]$, and

$$
\begin{aligned}
x(t)=T_{L}(t) L \phi(0) & +\int_{0}^{t}(t-s)^{\alpha-1} S_{L}(t-s) f\left(s, x_{s}\right) d s \\
& +\int_{0}^{t}(t-s)^{\alpha-1} S_{L}(t-s) \sigma\left(s, x_{s}\right) d \omega(t) \\
& +\sum_{0<t_{k}<t} T_{\alpha}\left(t-t_{k}\right) I_{k}\left(x\left(t_{k}^{-}\right)\right), t \in J .
\end{aligned}
$$

Set $\mathcal{B}_{T}^{0}=\left\{z \in \mathcal{B}_{T}\right.$, such that $\left.z_{0}=0\right\}$ and for any $z \in \mathcal{B}_{T}^{0}$ we have

$$
\|z\|_{\mathcal{B}_{T}^{0}}=\left\|z_{0}\right\|_{\mathcal{B}_{h}}+\sup _{t \in J}\left(E\|z(t)\|^{2}\right)^{\frac{1}{2}}=\sup _{t \in J}\left(E\|z(t)\|^{2}\right)^{\frac{1}{2}},
$$

where $\left\|z_{0}\right\|_{\mathcal{B}_{h}}=0$. Thus $\left(\mathcal{B}_{T}^{0},\|\cdot\|_{\mathcal{B}_{T}^{0}}\right)$ is a Banach space.

Let the operator $\widehat{\Psi}: \mathcal{B}_{T}^{0} \longrightarrow \mathcal{B}_{T}^{0}$ be defined by

$$
\widehat{\Psi} y(t)=\left\{\begin{array}{l}
0, \quad t \in(-\infty, 0] \\
\int_{0}^{t}(t-s)^{\alpha-1} S_{L}(t-s) f\left(s, y_{s}+\widehat{\phi}_{s}\right) d s \\
+\int_{0}^{t}(t-s)^{\alpha-1} S_{L}(t-s) \sigma\left(s, y_{s}+\widehat{\phi}_{s}\right) d \omega(t) \\
+\sum_{0<t_{k}<t} T_{L}\left(t-t_{k}\right) I_{k}\left(y\left(t_{k}^{-}\right)+\widehat{\phi}\left(t_{k}^{-}\right)\right), t \in J .
\end{array}\right.
$$


Set $\mathcal{B}_{r}=\left\{y \in \mathcal{B}_{T}^{0}, \quad\|y\|_{\mathcal{B}_{T}}^{2} \leq r, r>0\right\}$. The set $\mathcal{B}_{r}$ is clearly a bounded closed convex set in $\mathcal{B}_{T}^{0}$ for each $r>0$ and $y \in \mathcal{B}_{r}$, by Lemma 2.2 we have

$$
\begin{aligned}
\left\|y_{t}+\widehat{\phi}_{t}\right\|_{\mathcal{B}_{h}}^{2} \leq & 2\left(\left\|y_{t}\right\|_{\mathcal{B}_{h}}^{2}+\left\|\widehat{\phi}_{t}\right\|_{\mathcal{B}_{h}}^{2}\right) \\
& \leq 4\left(l^{2} \sup _{s \in[0, t]} E\|y(s)\|_{\mathcal{H}}^{2}+\left\|y_{0}\right\|_{\mathcal{B}_{h}}^{2}\right) \\
& +4\left(l^{2} \sup _{s \in[0, t]} E\|\widehat{\phi}(s)\|_{\mathcal{H}}^{2}+\left\|\widehat{\phi}_{0}\right\|_{\mathcal{B}_{h}}^{2}\right) \\
& \leq 4\|\phi\|_{\mathcal{B}_{h}}^{2}+4 l^{2}\left(r+M^{2} C_{1}^{2} C_{2}^{2} E\|\phi(0)\|_{\mathcal{H}}^{2}\right)=\chi .
\end{aligned}
$$

Now, let the two operators $\widehat{\Psi}_{1}$ and $\widehat{\Psi}_{2}$ be defined as

$$
\widehat{\Psi}_{1} y(t)=\left\{\begin{array}{lc}
0, & t \in(-\infty, 0], \\
\sum_{0<t_{k}<t} T_{L}\left(t-t_{k}\right) I_{k}\left(y\left(t_{k}^{-}\right)+\widehat{\phi}\left(t_{k}^{-}\right)\right), & t \in[0, T],
\end{array}\right.
$$

and

$$
\left(\widehat{\Psi}_{2} y\right)(t)= \begin{cases}0, & t \in(-\infty, 0], \\ \int_{0}^{t}(t-s)^{\alpha-1} S_{L}(t-s) f\left(s, y_{s}+\widehat{\phi}_{s}\right) d s & \\ +\int_{0}^{t}(t-s)^{\alpha-1} S_{L}(t-s) \sigma\left(s, y_{s}+\widehat{\phi}_{s}\right) d \omega(t), & t \in[0, T] .\end{cases}
$$

It is clear that

$$
\widehat{\Psi}_{1}+\widehat{\Psi}_{2}=\Psi
$$

Then, the problem of finding a solution of (2.1) is reduced to finding a solution of the operator equation $y(t)=\widehat{\Psi}_{1}(y)(t)+\widehat{\Psi}_{2}(y)(t), t \in(-\infty, T]$. In order to use Theorem 2.8 , we will verify that $\widehat{\Psi}_{1}$ is compact and continuous while $\widehat{\Psi}_{2}$ is a contraction operator.

For the sake of convenience, we divide the proof into several steps.

Step 1. We show that $\widehat{\Psi}_{1} y+\widehat{\Psi}_{2} y^{*} \in \mathcal{B}_{r}$, for $y, y^{*} \in \mathcal{B}_{r}$. For $t \in[0, T]$, we have 


$$
\begin{aligned}
\left\|\left(\widehat{\Psi}_{1} y\right)(t)+\left(\widehat{\Psi}_{2} y^{*}\right)(t)\right\|_{\mathcal{H}}^{2} \leq & 3 \sum_{0<t_{k}<t}\left\|T_{L}\left(t-t_{k}\right)\right\|^{2} E\left\|I_{k}\left(y\left(t_{k}^{-}\right)+\widehat{\phi}\left(t_{k}^{-}\right)\right)\right\|_{\mathcal{H}}^{2} \\
& +3 E\left\|\int_{0}^{t}(t-s)^{\alpha-1} S_{L}(t-s) f\left(s, y_{s}^{*}+\widehat{\phi}_{s}\right) d s\right\|_{\mathcal{H}}^{2} \\
& +3 E\left\|\int_{0}^{t}(t-s)^{\alpha-1} S_{L}(t-s) \sigma\left(s, y_{s}^{*}+\widehat{\phi}_{s}\right) d W(t)\right\|_{\mathcal{H}}^{2} \\
\leq & 3 M^{2} C_{1}^{2} \sum_{k=1}^{m} L_{k} \\
& +3 \int_{0}^{t}\left\|(t-s)^{\alpha-1} S_{L}(t-s)\right\|^{2} E\left\|f\left(s, y_{s}^{*}+\widehat{\phi}_{s}\right)\right\|_{\mathcal{H}}^{2} d s \\
& +3 \int_{0}^{t}\left\|(t-s)^{\alpha-1} S_{\alpha}(t-s)\right\|^{2} E\left\|\sigma\left(s, y_{s}^{*}+\widehat{\phi}_{s}\right)\right\|_{\mathcal{H}}^{2} d s \\
\leq & \frac{3 M^{2} C_{1}^{2}}{\Gamma^{2}(\alpha)} \frac{T^{\alpha}}{\alpha} \\
& \int_{0}^{t}(t-s)^{\alpha-1} d s+\frac{2 M^{2} C_{1}^{2} L_{\sigma} \chi}{\Gamma^{2}(\alpha)} \int_{0}^{t}(t-s)^{2(\alpha-1)} d s \\
\leq & 3 M^{2} C_{1}^{2} \sum_{k=1}^{m} L_{k}+\frac{3 M^{2} C_{1}^{2} L_{f} \chi}{\Gamma^{2}(\alpha)} \frac{T^{2 \alpha}}{\alpha^{2}}+\frac{2 M^{2} C_{1}^{2} L_{\sigma} \chi}{\Gamma^{2}(\alpha)} \frac{T^{2 \alpha-1}}{2 \alpha-1} \\
= & 3 M^{2} C_{1}^{2} \sum_{k=1}^{m} L_{k}+\frac{3 M^{2} C_{1}^{2} T^{2 \alpha} \chi}{\Gamma^{2}(\alpha)}\left[\frac{L_{f}}{\alpha^{2}}+\frac{L_{\sigma}}{T(2 \alpha-1)}\right]
\end{aligned}
$$

Then

$$
\left\|\left(\widehat{\Psi}_{1} y\right)(t)+\left(\widehat{\Psi}_{2} y^{*}\right)(t)\right\|_{\mathcal{H}}^{2} \leq 3 M^{2} C_{1}^{2} \sum_{k=1}^{m} L_{k}+\frac{3 M^{2} C_{1}^{2} T^{2 \alpha} \chi}{\Gamma^{2}(\alpha)}\left[\frac{L_{f}}{\alpha^{2}}+\frac{L_{\sigma}}{T(2 \alpha-1)}\right] \leq r .
$$

Hence, we get $\widehat{\Psi}_{1} y+\widehat{\Psi}_{2} y^{*} \in \mathcal{B}_{r}$.

Step 2. The map $\widehat{\Psi}_{1}$ is continuous on $\mathcal{B}_{r}$.

Let $\left\{y^{n}\right\}_{n=1}^{\infty}$ be a sequence in $\mathcal{B}_{r}$ with $\lim y^{n} \rightarrow y \in \mathcal{B}_{r}$. Then for $t \in J$ we have

$$
\begin{gathered}
E\left\|\left(\widehat{\Psi}_{1} y^{n}\right)(t)-\left(\widehat{\Psi}_{1} y\right)(t)\right\|_{\mathcal{H}}^{2} \leq \\
\sum_{0<t_{k}<t}\left\|T_{\alpha}\left(t-t_{k}\right)\right\|^{2} E\left\|I_{k}\left(y^{n}\left(t_{k}^{-}\right)+\widehat{\phi}\left(t_{k}^{-}\right)\right)-I_{k}\left(y\left(t_{k}^{-}\right)+\widehat{\phi}\left(t_{k}^{-}\right)\right)\right\|_{\mathcal{H}}^{2} .
\end{gathered}
$$

Since the functions $I_{i}, i=1,2, \ldots, m$ are continuous hence $\lim _{n \rightarrow \infty} \|\left(\widehat{\Psi}_{1} y^{n}\right)(t)-$ $\left(\widehat{\Psi}_{1} y\right)(t) \|_{\mathcal{H}}^{2}=0$ which implies that the mapping $\widehat{\Psi}_{1}$ is continuous on $\mathcal{B}_{r}$. 
Step 3. $\widehat{\Psi}_{1}$ maps bounded sets into bounded sets in $\mathcal{B}_{r}$.

Let us prove that for $r>0$ there exists a $\widehat{r}>0$ such that for each $y \in \mathcal{B}_{r}$ we have $E\left\|\left(\widehat{\Psi}_{1} y\right)(t)\right\|_{\mathcal{H}}^{2}<\widehat{r}$ for $t \in J$.

$$
\begin{aligned}
E\left\|\left(\widehat{\Psi}_{1} y\right)(t)\right\|_{\mathcal{H}}^{2} & \leq \sum_{0<t_{k}<t}\left\|T_{L}\left(t-t_{k}\right)\right\|^{2} E\left\|I_{k}\left(y\left(t_{k}^{-}\right)+\widehat{\phi}\left(t_{k}^{-}\right)\right)\right\|_{\mathcal{H}}^{2} \\
& \leq M^{2} C_{1}^{2} \sum_{0}^{m} L_{k}=\widehat{r}
\end{aligned}
$$

which proves the desired result.

Step 4. The map $\widehat{\Psi}_{1}$ is equicontinuous.

Let $u, v \in J, 0 \leq u<v \leq T, y \in \mathcal{B}_{r}$, we obtain

$$
E\left\|\left(\widehat{\Psi}_{1} y\right)(v)-\left(\widehat{\Psi}_{1} y\right)(u)\right\|_{\mathcal{H}}^{2} \leq C_{1}^{2} \sum_{0<t_{k}<u} L_{k}\left\|T_{\alpha}\left(v-t_{k}\right)-T_{\alpha}\left(u-t_{k}\right)\right\|^{2} .
$$

The right-hand side tends to zero as $v-u \longrightarrow 0$, since $T_{\alpha}$ is strongly continuous and it allows us to conclude that

$$
\lim _{u \rightarrow v}\left\|T_{\alpha}\left(v-t_{k}\right)-T_{\alpha}\left(u-t_{k}\right)\right\|^{2}=0,
$$

which implies that $\widehat{\Psi}_{1}\left(\mathcal{B}_{r}\right)$ is equicontinuous.

Finally, combining Step 1 to Step 4 together with Ascoli's theorem, we conclude that the operator $\widehat{\Psi}_{1}$ is compact.

Step 5. $\widehat{\Psi}_{2}$ is a contraction mapping.

Let $y, y^{*} \in \mathcal{B}_{r}$ and $t \in J$ we have

$$
\begin{aligned}
E \|\left(\widehat{\Psi}_{2} y\right)(t)- & \left(\widehat{\Psi}_{2} y^{*}\right)(t) \|_{\mathcal{H}}^{2} \\
\leq & 2 E\left\|\int_{0}^{t}(t-s)^{\alpha-1} S_{L}(t-s)\left[f\left(s, y_{s}+\widehat{\phi}_{s}\right)-f\left(s, y_{s}^{*}+\widehat{\phi}_{s}\right)\right] d s\right\|_{\mathcal{H}}^{2} \\
& +2 E\left\|\int_{0}^{t}(t-s)^{\alpha-1} S_{L}(t-s)\left[\sigma\left(s, y_{s}+\widehat{\phi}_{s}\right)-\sigma\left(s, y_{s}^{*}+\widehat{\phi}_{s}\right)\right] d \omega(s)\right\|_{\mathcal{H}}^{2} \\
\leq & 2 \int_{0}^{t}\left\|(t-s)^{\alpha-1} S_{L}(t-s)\right\| d s \int_{0}^{t}\left\|(t-s)^{\alpha-1} S_{L}(t-s)\right\| \\
& \times E\left\|f\left(s, y_{s}+\widehat{\phi}_{s}\right)-f\left(s, y_{s}^{*}+\widehat{\phi}_{s}\right)\right\|_{\mathcal{H}}^{2} d s \\
& +2 \int_{0}^{t}\left\|(t-s)^{\alpha-1} S_{L}(t-s)\right\|^{2} E\left\|\sigma\left(s, y_{s}+\widehat{\phi}_{s}\right)-\sigma\left(s, y_{s}^{*}+\widehat{\phi}_{s}\right)\right\|_{\mathcal{H}}^{2} d s
\end{aligned}
$$




$$
\begin{aligned}
\leq & 2 \frac{M^{2} C_{1}^{2}}{\Gamma^{2}(\alpha)} \int_{0}^{t}(t-s)^{\alpha-1} d s \int_{0}^{t}(t-s)^{\alpha-1} L_{f}\left\|y(s)-y^{*}(s)\right\|_{\mathcal{B}_{h}}^{2} d s \\
& +\frac{2 M^{2} C_{1}^{2}}{\Gamma^{2}(\alpha)} \int_{0}^{t}(t-s)^{2(\alpha-1)} L_{\sigma}\left\|y_{s}-y_{s}^{*}\right\|_{\mathcal{B}_{h}}^{2} d s \\
\leq & \frac{2 M^{2} C_{1}^{2}}{\Gamma^{2}(\alpha)} T^{2 \alpha}\left[\frac{L_{f} l}{\alpha^{2}}+\frac{L_{\sigma} l}{T(2 \alpha-1)}\right]\left\|y-y^{*}\right\|_{\mathcal{B}_{T}^{0}}^{2} .
\end{aligned}
$$

By the condition (3.3), we obtain that $\widehat{\Psi}_{2}$ is a contraction mapping. Hence, by Krasnoselskii's fixed point theorem we can conclude that the problem (2.1) has at least one solution on $(-\infty, T]$. This completes the proof of the theorem.

Example 3.3. In this section, we consider an example to illustrate our main theorem. We examine the existence of solutions for the following fractional stochastic partial differential equation of the form

$$
\left\{\begin{aligned}
& D_{t}^{\alpha}\left[z(t, x)-z_{x x}(t, x)\right]=z_{x x}(t, x)+F(t, z(t-R, x)) \\
&+G(t, z(t-R, x)) \frac{d \omega(t)}{d t}, x \in=[0, \pi], R>0, t \neq t_{k} \\
& I_{k}\left(z\left(t_{k}^{-}, x\right)\right)=z\left(t_{k}^{+}, x\right)-z\left(t_{k}^{-}, x\right), \quad k=1, \ldots, n \\
& z(t, x)=\phi(t, x), \quad t \in(-\infty, 0], x \in[0, \pi] \\
& z(t, 0)=0=z(t, \pi), t \geq 0
\end{aligned}\right.
$$

where $\omega(t)$ is a standard cylindrical Wiener process in $\mathcal{H}$ defined on a stochastic space $\left(\Omega, \mathcal{F}, \mathbb{P},\left\{\mathcal{F}_{t}\right\}\right) ; D_{t}^{\alpha}$ is the Caputo fractional derivative of order $0<\alpha<1$; $0<t_{1}<t_{2}<\cdots<t_{n}<T$ are prefixed numbers.

Let $\mathcal{K}=\mathcal{H}=L^{2}([0, \pi])$ with the norm $\|\cdot\|$. Define the operators $A: D(A) \subset$ $\mathcal{H} \longrightarrow \mathcal{H}, L: D(L) \subset \mathcal{H} \longrightarrow \mathcal{H}$, by $A z=-z^{\prime \prime}$ and $L z=z-z^{\prime \prime}$, where each domain $D(A)$ and $D(L)$ is given by $\left\{z \in \mathcal{H}, z, z^{\prime}\right.$ are absolutely continuous, $z^{\prime \prime} \in$ $\mathcal{H}$ and $z(0)=z(\pi)=0\}$.

Further, $A$ and $L$ can be $A z=\sum_{n=1}^{\infty} n^{2}\left(z, z_{n}\right) z_{n}, z \in D(A), L z=\sum_{n=1}^{\infty}\left(1+n^{2}\right)\left(z, z_{n}\right) z_{n}$, $z \in D(L)$, where $z_{n}(x)=\sqrt{\frac{2}{\pi}} \sin (n x), n=1,2, \ldots$, is the orthogonal set of vectors of $A$. Also, for $z \in \mathcal{H} L^{-1} z=\sum_{n=1}^{\infty} \frac{1}{\left(1+n^{2}\right)}\left(z, z_{n}\right) z_{n}, A L^{-1} z=\sum_{n=1}^{\infty} \frac{n}{\left(1+n^{2}\right)}\left(z, z_{n}\right) z_{n}$, $T(t) z=\sum_{n=1}^{\infty} \exp \frac{n^{2} t}{\left(1+n^{2}\right)}\left(z, z_{n}\right) z_{n}$

It is easy to see that $-A L^{-1}$ generates a uniformly continuous semigroup $T(t), t \geq 0$ and so $\max _{t \in[0, T]}\|T(t)\|$ is finite. 
Let $h(t)=e^{2 t}, t<0$, then $l=\int_{-\infty}^{0} \frac{h(s)}{s} d s=\frac{1}{2}$ and define

$$
\|\phi\|_{\mathcal{B}_{h}}=\int_{-\infty}^{0} h(s) \sup _{s \leq \theta \leq 0}\left(E|\theta|^{2}\right)^{\frac{1}{2}} d s .
$$

Hence for $(t, \phi) \in[0, T] \times \mathcal{B}_{h}$, where $\phi(\theta)(x)=\phi(\theta, x),(\theta, x) \in(-\infty, 0] \times[0, \pi]$. But $z(t)=z(t, \cdot)$, that is $z(t)(x)=z(t, x)$. Define $f:[0, T] \times \mathcal{B}_{h} \longrightarrow L^{2}([0, \pi])$ and $\sigma:[0, T] \times \mathcal{B}_{h} \longrightarrow \mathcal{L}_{2}^{0}$ as follows:

$$
\begin{aligned}
& f(t, \phi) x=F(t, x(\cdot)), \\
& \sigma(t, \phi) x=G(t, x(\cdot)) .
\end{aligned}
$$

With the choice of $A, f$ and $\sigma$ can be rewritten as the abstract form of system (2.1). Under the appropriate conditions on the functions $f, \sigma$ and $I_{k}$ as those in (H1)-(H3), system (3.6) has a mild solution on $(-\infty, T]$.

\section{Controllability results}

In this section, we treat the controllability of Sobolev-type impulsive stochastic fractional equations with nonlocal conditions using the argument of the previous section. More precisely we will consider the following problem:

$$
\left\{\begin{array}{l}
\left.D_{t}^{\alpha} L x(t)=A x(t)+B u(t)+f\left(t, x_{t}\right)\right)+\sigma\left(t, x_{t}\right) \frac{d \omega(t)}{d t}, \quad t \in J=[0, T], T>0, t \neq t_{k} \\
\Delta x\left(t_{k}\right)=I_{k}\left(x\left(t_{k}^{-}\right)\right), \quad k=1, \ldots, m \\
x(0)+g(x)=x_{0}=\phi, \quad \phi \in B_{h},
\end{array}\right.
$$

where $A, L, f, \sigma$ and $I_{k}$ are as in Section 3 , the nonlocal function $g: \mathcal{B}_{h} \longrightarrow \mathcal{H}$. The control function $u(\cdot)$ is given in $L^{2}(J, \mathcal{U})$ a Banach space of admissible control functions for a separable Hilbert space $\mathcal{U}$. Finally $B$ is a bounded linear operator from $\mathcal{U}$ to $\mathcal{H}$.

Definition 4.1. An $\mathcal{F}_{t}$-adapted stochastic process $x:(-\infty, T] \longrightarrow \mathcal{H}$ is said to be a mild solution of $(4.1)$ if $x_{0}=\phi(t)$ on $(-\infty, 0]$ :

1. $x(t)$ is $\mathcal{B}_{h}$-valued and the restriction of $x(\cdot)$ to $\left(t_{k}, t_{k+1}\right], k=1,2, \ldots, m$ is continuous.

2. for each $t \in J, x(t)$ satisfies the following integral equation:

$$
\begin{aligned}
x(t)=T_{L}(t) L(\phi(0)-g(x)) & +\int_{0}^{t}(t-s)^{\alpha-1} S_{L}(t-s) B u(s) d s \\
& +\int_{0}^{t}(t-s)^{\alpha-1} S_{L}(t-s) f\left(s, x_{s}\right) d s \\
& +\int_{0}^{t}(t-s)^{\alpha-1} S_{L}(t-s) \sigma\left(s, x_{s}\right) d \omega(t) \\
& +\sum_{0<t_{k}<t} T_{L}\left(t-t_{k}\right) I_{k}\left(x\left(t_{k}^{-}\right)\right),
\end{aligned}
$$




$$
\mathrm{P} \text { - a.s for all } t \in J \text {. }
$$

Definition 4.2. The system (4.1) is said to be controllable on the interval $(-\infty, T]$ if for every initial value $\phi$ and every $x_{1} \in \mathcal{H}$, there exists a control $u \in L^{2}(J, \mathcal{U})$, such that the mild solution $x(t)$ of system (4.1) satisfies $y(T)=x_{1}$.

Our main result in this section is the following. We shall assume some additional hypotheses:

(H4) The linear operator $W$ from $L^{2}(J, \mathcal{U})$ into $\mathcal{H}$ defined by

$$
W u=\int_{0}^{T}(T-s)^{\alpha-1} S_{L}(T-s) B u(s) d s
$$

has an induced inverse $W^{-1}$ which takes values in $L^{2}(J, \mathcal{U}) k e r W$, and there exist positive constants $M_{1}, M_{2}$ such that $\|B\|^{2}=M_{1},\left\|W^{-1}\right\|^{2}=M_{2}$.

(H5) There exists $L_{g}>0$ such that

$$
E\|g(x)-g(y)\|_{\mathcal{H}}^{2} \leq L_{g}\|x-y\|_{\mathcal{B}_{h}}^{2}, \quad t \geq 0 .
$$

Theorem 4.3. Assume that $f(t, 0)=\sigma(t, 0)=g(0)=0, \forall t \geq 0$. Assume that hypotheses $(H 1)-(H 3)$ and $(H 4)-(H 5)$ hold. If

$$
r^{*} \geq 5 M^{2} C_{1}^{2}\left(\sum_{k=1}^{m} L_{k}+C_{2}^{2} L_{g} \chi^{*}\right)+\frac{5 M^{2} C_{1}^{2} T^{2 \alpha}}{\Gamma^{2}(\alpha)}\left[\frac{\chi^{*} L_{f}}{\alpha^{2}}+\frac{\chi^{*} L_{\sigma}}{T(2 \alpha-1)}+\frac{\xi M_{1}}{T(2 \alpha-1)}\right]
$$

and

$$
\begin{gathered}
\Lambda=4 M^{2} C_{1}^{2} C_{2}^{2} L_{g} l+4 \frac{M^{2} C_{1}^{2}}{\Gamma^{2}(\alpha)} T^{2 \alpha}\left[\frac{L_{f} l}{\alpha^{2}}\left(1+\frac{3 M_{1} M_{2} M^{2} C_{1}^{2} T^{2 \alpha}}{\alpha^{2} \Gamma^{2}(\alpha)}\right)\right. \\
\left.+\frac{L_{\sigma} l}{T(2 \alpha-1)}\left(1+\frac{3 M_{1} M_{2} M^{2} C_{1}^{2} T^{2 \alpha}}{T(2 \alpha-1) \alpha^{2} \Gamma^{2}(\alpha)}\right)\right]<1
\end{gathered}
$$

then the system (4.1) is controllable on $(-\infty, T]$.

Proof. Using assumption $(\mathrm{H} 4)$, for an arbitrary process $x(\cdot)$, define the control process

$$
\begin{aligned}
u_{x}(t)= & W^{-1}\left\{x_{1}-T_{L}(t) L(\phi(0)-g(x))-\int_{0}^{t}(t-s)^{\alpha-1} S_{L}(t-s) f\left(s, x_{s}\right) d s\right. \\
& -\int_{0}^{t}(t-s)^{\alpha-1} S_{L}(t-s) \sigma\left(s, x_{s}\right) d \omega(t) \\
& \left.-\sum_{0<t_{k}<t} T_{L}\left(t-t_{k}\right) I_{k}\left(x\left(t_{k}^{-}\right)\right)\right\}(t) .
\end{aligned}
$$

It shall now be shown that when using this control, the operator $\Psi^{*}$ defined by 
$\Psi^{*} x(t)=\left\{\begin{array}{l}\phi(t), \quad \text { if } \quad t \in(-\infty, 0] \\ T_{L}(t) L(\phi(0)-g(x))+\int_{0}^{t}(t-s)^{\alpha-1} S_{L}(t-s) B u(s) d s \\ +\int_{0}^{t}(t-s)^{\alpha-1} S_{L}(t-s) f\left(s, x_{s}\right) d s+\int_{0}^{t}(t-s)^{\alpha-1} S_{L}(t-s) \sigma\left(s, x_{s}\right) d \omega(t) \\ +\sum_{0<t_{k}<t} T_{L}\left(t-t_{k}\right) I_{k}\left(x\left(t_{k}^{-}\right)\right) \text {for all } t \in J,\end{array}\right.$

from $\mathcal{B}_{T}$ into itself for each $y \in \mathcal{B}_{T}$ has a fixed point. This fixed point is then a solution of equation (4.1).

For $\phi \in \mathcal{B}_{h}$, we define $\widehat{\phi}$ by

$$
\widehat{\phi}(t)=\left\{\begin{array}{cc}
\phi(t), & t \in(-\infty, 0], \\
T_{L}(t) L \phi(0), & t \in[0,+\infty[;
\end{array} \text { then } \widehat{\phi} \in \mathcal{B}_{T} .\right.
$$

Let $x(t)=y(t)+\widehat{\phi}(t),-\infty<t<T$.

It is evident that $y$ satisfies $y_{0}=0, t \in(-\infty, 0]$, and

$$
\begin{aligned}
y(t)= & -T_{L}(t) L g(y+\widehat{\phi})+\int_{0}^{t}(t-s)^{\alpha-1} S_{L}(t-s) B u_{y+\widehat{\phi}}(s) d s \\
& +\int_{0}^{t}(t-s)^{\alpha-1} S_{L}(t-s) f\left(s, y_{s}+\widehat{\phi}_{s}\right) d s \\
& +\int_{0}^{t}(t-s)^{\alpha-1} S_{L}(t-s) \sigma\left(s, y_{s}+\widehat{\phi}_{s}\right) d \omega(t) \\
& +\sum_{0<t_{k}<t} T_{\alpha}\left(t-t_{k}\right) I_{k}\left(y\left(t_{k}^{-}\right)+\widehat{\phi}\left(t_{k}^{-}\right)\right), t \in J,
\end{aligned}
$$

where $u_{y+\widehat{\phi}}$ is obtained from (4.5) by replacing $x_{t}$ by $y_{t}+\widehat{\phi}_{t}$.

Set $\mathcal{B}_{T}^{0}=\left\{z \in \mathcal{B}_{T}\right.$, such that $\left.z_{0}=0\right\}$ and for any $z \in \mathcal{B}_{T}^{0}$ we have

$$
\|z\|_{\mathcal{B}_{T}^{0}}=\left\|z_{0}\right\|_{\mathcal{B}_{h}}+\sup _{t \in J}\left(E\|z(t)\|^{2}\right)^{\frac{1}{2}}=\sup _{t \in J}\left(E\|z(t)\|^{2}\right)^{\frac{1}{2}},
$$

where $\left\|z_{0}\right\|_{\mathcal{B}_{h}}=0$. Thus $\left(\mathcal{B}_{T}^{0},\|\cdot\|_{\mathcal{B}_{T}^{0}}\right)$ is a Banach space.

Let the operator $\widehat{\Psi}^{*}: \mathcal{B}_{T}^{0} \longrightarrow \mathcal{B}_{T}^{0}$ be defined by

$$
\widehat{\Psi}^{*} y(t)=\left\{\begin{array}{l}
0, \quad t \in(-\infty, 0] \\
-T_{L}(t) L g(y+\widehat{\phi})+\int_{0}^{t}(t-s)^{\alpha-1} S_{L}(t-s) B u_{y+\widehat{\phi}}(s) d s \\
+\int_{0}^{t}(t-s)^{\alpha-1} S_{L}(t-s) f\left(s, y_{s}+\widehat{\phi}_{s}\right) d s \\
+\int_{0}^{t}(t-s)^{\alpha-1} S_{L}(t-s) \sigma\left(s, y_{s}+\widehat{\phi}_{s}\right) d \omega(t) \\
+\sum_{0<t_{k}<t} T_{L}\left(t-t_{k}\right) I_{k}\left(y\left(t_{k}^{-}\right)+\widehat{\phi}\left(t_{k}^{-}\right)\right), t \in J .
\end{array}\right.
$$


Set $\mathcal{B}_{r^{*}}=\left\{y \in \mathcal{B}_{T}^{0}, \quad\|y\|_{\mathcal{B}_{T}^{0}}^{2} \leq r^{*}, r^{*}>0\right\}$. The set $\mathcal{B}_{r^{*}}$ is clearly a bounded closed convex set in $\mathcal{B}_{T}^{0}$ for each $r^{*}>0$ and for each $y \in \mathcal{B}_{r^{*}}$. By Lemma 2.2 we have

$$
\begin{aligned}
\left\|y_{t}+\widehat{\phi}_{t}\right\|_{\mathcal{B}_{h}}^{2} \leq & 2\left(\left\|y_{t}\right\|_{\mathcal{B}_{h}}^{2}+\left\|\widehat{\phi}_{t}\right\|_{\mathcal{B}_{h}}^{2}\right) \\
& \leq 4\left(l^{2} \sup _{s \in[0, t]} E\|y(s)\|_{\mathcal{H}}^{2}+\left\|y_{0}\right\|_{\mathcal{B}_{h}}^{2}\right) \\
& +4\left(l^{2} \sup _{s \in[0, t]} E\|\widehat{\phi}(s)\|_{\mathcal{H}}^{2}+\left\|\widehat{\phi}_{0}\right\|_{\mathcal{B}_{h}}^{2}\right) \\
& \leq 4\|\phi\|_{\mathcal{B}_{h}}^{2}+4 l^{2}\left(r^{*}+M^{2} C_{1}^{2} C_{2}^{2} E\|\phi(0)\|_{\mathcal{H}}^{2}\right)=\chi^{*} .
\end{aligned}
$$

Now, let the two operators $\widehat{\Psi}_{1}^{*}$ and $\widehat{\Psi}_{2}^{*}$ defined as

$$
\widehat{\Psi}_{1}^{*} y(t)=\left\{\begin{array}{lc}
0 & t \in(-\infty, 0] \\
\sum_{0<t_{k}<t} T_{L}\left(t-t_{k}\right) I_{k}\left(y\left(t_{k}^{-}\right)+\widehat{\phi}\left(t_{k}^{-}\right)\right), & t \in[0, T]
\end{array}\right.
$$

and

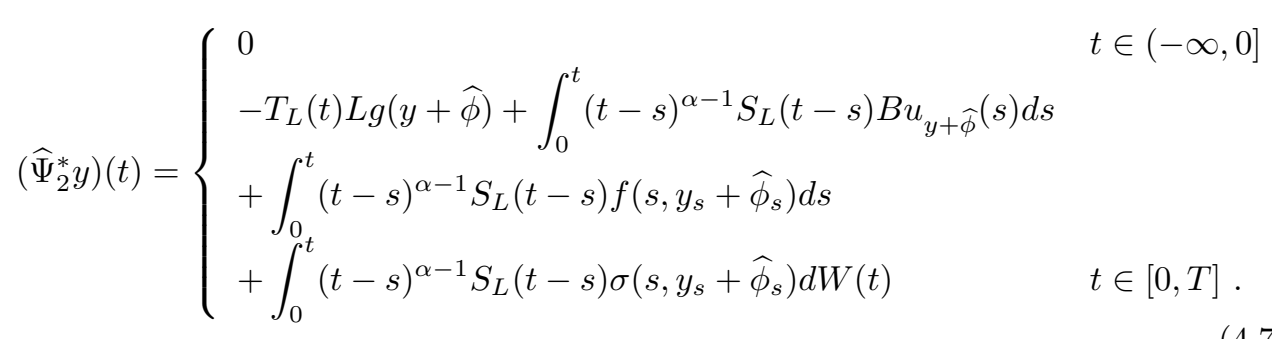

It is clear that

$$
\widehat{\Psi}_{1}^{*}+\widehat{\Psi}_{2}^{*}=\Psi^{*}
$$

Then, the problem of finding a solution of (4.1) is reduced to finding a solution of the operator equation $y(t)=\widehat{\Psi}_{1}^{*}(y)(t)+\widehat{\Psi}_{2}^{*}(y)(t), t \in(-\infty, T]$. In order to use Theorem 2.8 we will verify that $\widehat{\Psi}_{1}^{*}$ is compact and continuous while $\widehat{\Psi}_{2}^{*}$ is a contraction operator.

For the sake of convenience, we divide the proof into several steps. 
Step 1. We show that $\widehat{\Psi}_{1}^{*} y+\widehat{\Psi}_{2}^{*} y^{*} \in \mathcal{B}_{r^{*}}$, for $y, y^{*} \in \mathcal{B}_{r^{*}}$. For $t \in[0, T]$, we have

$$
\begin{aligned}
\left\|\left(\widehat{\Psi}_{1} y\right)(t)+\left(\widehat{\Psi}_{2} y^{*}\right)(t)\right\|_{\mathcal{H}}^{2} \leq & 5 \sum_{0<t_{k}<t}\left\|T_{L}\left(t-t_{k}\right)\right\|^{2} E\left\|I_{k}\left(y\left(t_{k}^{-}\right)+\widehat{\phi}\left(t_{k}^{-}\right)\right)\right\|_{\mathcal{H}}^{2} \\
& +5\left\|T_{L}(t) L\right\|^{2} E\|g(y+\widehat{\phi})\|_{\mathcal{H}}^{2} \\
& +5 E\left\|\int_{0}^{t}(t-s)^{\alpha-1} S_{L}(t-s) f\left(s, y_{s}^{*}+\widehat{\phi}_{s}\right) d s\right\|_{\mathcal{H}}^{2} \\
& +5 E\left\|\int_{0}^{t}(t-s)^{\alpha-1} S_{L}(t-s) \sigma\left(s, y_{s}^{*}+\widehat{\phi}_{s}\right) d \omega(t)\right\|_{\mathcal{H}}^{2} \\
& +5 \int_{0}^{t}(t-s)^{\alpha-1} S_{L}(t-s) B u_{y^{*}+\widehat{\phi}}(s) d s .
\end{aligned}
$$

Observe that

$$
\begin{aligned}
E\left\|u_{y^{*}+\widehat{\phi}}\right\|^{2} \leq & 6 M_{2}\left\{E\left|x_{1}\right|_{\mathcal{H}}^{2}+M^{2} C_{1}^{2} C_{2}^{2} E|\phi(0)|_{\mathcal{H}}^{2}+M^{2} C_{1}^{2} C_{2}^{2} L_{g} \chi^{*}+M^{2} C_{1}^{2} \sum_{k=1}^{m} L_{k}\right. \\
& \left.+\frac{M^{2} C_{1}^{2} T^{2 \alpha} \chi^{*}}{\Gamma^{2}(\alpha)}\left[\frac{L_{f}}{\alpha^{2}}+\frac{L_{\sigma}}{T(2 \alpha-1)}\right]\right\}=\xi .
\end{aligned}
$$

Then

$$
\begin{aligned}
\left\|\left(\widehat{\Psi}_{1} y\right)(t)+\left(\widehat{\Psi}_{2} y^{*}\right)(t)\right\|_{\mathcal{H}}^{2} \leq & 5 M^{2} C_{1}^{2} \sum_{k=1}^{m} L_{k}+5\left\|T_{L}(t) L\right\|^{2} E\left\|g\left(y^{*}+\widehat{\phi}\right)\right\|_{\mathcal{H}}^{2} \\
& +5 \int_{0}^{t}\left\|(t-s)^{\alpha-1} S_{L}(t-s)\right\|^{2} E\left\|f\left(s, y_{s}^{*}+\widehat{\phi}_{s}\right)\right\|_{\mathcal{H}}^{2} d s \\
& +5 \int_{0}^{t}\left\|(t-s)^{\alpha-1} S_{\alpha}(t-s)\right\|^{2} E\left\|\sigma\left(s, y_{s}^{*}+\widehat{\phi}_{s}\right)\right\|_{\mathcal{H}}^{2} d s \\
& +5 \int_{0}^{t}(t-\eta)^{\alpha-1} S_{L}(t-\eta) B u_{y^{*}+\widehat{\phi}}(\eta) d \eta \\
\leq & 5 M^{2} C_{1}^{2} \sum_{k=1}^{m} L_{k}+5 M^{2} C_{1}^{2} C_{2}^{2} L_{g} \chi^{*} \\
& +\frac{5 M^{2} C_{1}^{2} L_{f} \chi^{*}}{\Gamma^{2}(\alpha)} \frac{T^{\alpha}}{\alpha} \int_{0}^{t}(t-s)^{\alpha-1} d s \\
& +\frac{5 M^{2} C_{1}^{2} L_{\sigma} \chi^{*}}{\Gamma^{2}(\alpha)} \int_{0}^{t}(t-s)^{2(\alpha-1)} d s \\
& +\frac{5 M^{2} C_{1}^{2} M_{1} \xi}{\Gamma^{2}(\alpha)} \int_{0}^{t}(t-\eta)^{2(\alpha-1)} d \eta
\end{aligned}
$$




$$
\begin{aligned}
& \leq 5 M^{2} C_{1}^{2}\left(\sum_{k=1}^{m} L_{k}+C_{2}^{2} L_{g} \chi^{*}\right) \\
& +\frac{5 M^{2} C_{1}^{2} L_{f} \chi^{*}}{\Gamma^{2}(\alpha)} \frac{T^{2 \alpha}}{\alpha^{2}}+\frac{5 M^{2} C_{1}^{2} L_{\sigma} \chi^{*}}{\Gamma^{2}(\alpha)} \frac{T^{2 \alpha-1}}{2 \alpha-1} \\
& +\frac{5 M^{2} C_{1}^{2} M_{1} \xi}{\Gamma^{2}(\alpha)} \frac{T^{2 \alpha-1}}{2 \alpha-1} \\
= & 5 M^{2} C_{1}^{2}\left(\sum_{k=1}^{m} L_{k}+C_{2}^{2} L_{g} \chi^{*}\right) \\
& +\frac{5 M^{2} C_{1}^{2} T^{2 \alpha}}{\Gamma^{2}(\alpha)}\left[\frac{\chi^{*} L_{f}}{\alpha^{2}}+\frac{\chi^{*} L_{\sigma}}{T(2 \alpha-1)}+\frac{\xi M_{1}}{T(2 \alpha-1)}\right] .
\end{aligned}
$$

Then

$$
\begin{aligned}
\left\|\left(\Psi_{1} y\right)(t)+\left(\Psi_{2} y^{*}\right)(t)\right\|_{\mathcal{H}}^{2} & \leq 5 M^{2} C_{1}^{2}\left(\sum_{k=1}^{m} L_{k}+C_{2}^{2} L_{g} \chi^{*}\right) \\
& +\frac{5 M^{2} C_{1}^{2} T^{2 \alpha}}{\Gamma^{2}(\alpha)}\left[\frac{\chi^{*} L_{f}}{\alpha^{2}}+\frac{\chi^{*} L_{\sigma}}{T(2 \alpha-1)}+\frac{\xi M_{1}}{T(2 \alpha-1)}\right] \leq r^{*} .
\end{aligned}
$$

Hence, we get $\widehat{\Psi}_{1}^{*} y+\widehat{\Psi}_{2}^{*} y^{*} \in \mathcal{B}_{r}^{*}$.

Step 2. As in Section 3, we can prove that the operators $\widehat{\Psi}_{1}^{*}$ is compact and continuous.

Step 3. $\widehat{\Psi}_{2}^{*}$ is a contraction mapping.

Let $y, y^{*} \in \mathcal{B}_{r^{*}}$ and $t \in J$ we have

$$
\begin{aligned}
\|\left(\widehat{\Psi}_{2}^{*} y\right)(t) & -\left(\widehat{\Psi}_{2}^{*} y^{*}\right)(t)\left\|_{\mathcal{H}}^{2} \leq 4 E\right\| T_{L}(t) L\left[g(y+\widehat{\phi})-g\left(y^{*}+\widehat{\phi}\right)\right] \|_{\mathcal{H}}^{2} \\
& +4 E\left\|\int_{0}^{t}(t-s)^{\alpha-1} S_{L}(t-s)\left[f\left(s, y_{s}+\widehat{\phi}_{s}\right)-f\left(s, y_{s}^{*}+\widehat{\phi}_{s}\right)\right] d s\right\|_{\mathcal{H}}^{2} \\
& +4 E\left\|\int_{0}^{t}(t-s)^{\alpha-1} S_{L}(t-s)\left[\sigma\left(s, y_{s}+\widehat{\phi}_{s}\right)-\sigma\left(s, y_{s}^{*}+\widehat{\phi}_{s}\right)\right] d \omega(s)\right\|_{\mathcal{H}}^{2} \\
& +4 E\left\|\int_{0}^{t}(t-\eta)^{\alpha-1} S_{L}(t-\eta) B\left(u_{y+\widehat{\phi}}-u_{y^{*}+\widehat{\phi}}\right) d \eta\right\|_{\mathcal{H}}^{2}
\end{aligned}
$$




$$
\begin{aligned}
& \leq 4 M^{2} C_{1}^{2} C_{2}^{2} E\left\|g(y+\widehat{\phi})-g\left(y^{*}+\widehat{\phi}\right)\right\|_{\mathcal{H}}^{2} \\
& +4 \int_{0}^{t}\left\|(t-s)^{\alpha-1} S_{L}(t-s)\right\| d s \int_{0}^{t}\left\|(t-s)^{\alpha-1} S_{L}(t-s)\right\| \\
& \times E\left\|f\left(s, y_{s}+\widehat{\phi}_{s}\right)-f\left(s, y_{s}^{*}+\widehat{\phi}_{s}\right)\right\|_{\mathcal{H}}^{2} d s \\
& +4 \int_{0}^{t}\left\|(t-s)^{\alpha-1} S_{L}(t-s)\right\|^{2} E\left\|\sigma\left(s, y_{s}+\widehat{\phi}_{s}\right)-\sigma\left(s, y_{s}^{*}+\widehat{\phi}_{s}\right)\right\|_{L_{2}^{0}}^{2} d s \\
& +12 M_{1} M_{2} \int_{0}^{t}\left\|(t-\eta)^{\alpha-1} S_{L}(T-\eta)\right\| d \eta \int_{0}^{t}\left\|(t-\eta)^{\alpha-1} S_{L}(T-\eta)\right\| \\
& \times\left[\int_{0}^{t}\left\|(t-s)^{\alpha-1} S_{L}(t-s)\right\| d s \int_{0}^{t}\left\|(t-s)^{\alpha-1} S_{L}(t-s)\right\|\right. \\
& \left.\times E\left\|f\left(s, y_{s}+\widehat{\phi}_{s}\right)-f\left(s, y_{s}^{*}+\widehat{\phi}_{s}\right)\right\|_{\mathbb{H}}^{2} d s\right] d \eta \\
& +12 M_{1} M_{2} \int_{0}^{t}\left\|(t-\eta)^{\alpha-1} S_{L}(T-\eta)\right\|^{2}\left[\int_{0}^{t}\left\|(t-s)^{\alpha-1} S_{L}(t-s)\right\|^{2}\right. \\
& \left.\times E\left\|\sigma\left(s, y_{s}+\widehat{\phi}_{s}\right)-\sigma\left(s, y_{s}^{*}+\widehat{\phi}_{s}\right)\right\|_{\mathcal{H}}^{2} d s\right] d \eta \\
& +12 M_{1} M_{2} M^{2} C_{1}^{2} C_{2}^{2} E\left\|g(y+\widehat{\phi})-g\left(y^{*}+\widehat{\phi}\right)\right\|_{\mathcal{H}}^{2} \\
& \leq 4 M^{2} C_{1}^{2} C_{2}^{2} L_{g}\left\|y-y^{*}\right\|_{\mathcal{B}_{h}}^{2} \\
& +\frac{4 M^{2} C_{1}^{2}}{\Gamma^{2}(\alpha)} \int_{0}^{t}(t-s)^{\alpha-1} d s \int_{0}^{t}(t-s)^{\alpha-1} L_{f}\left\|y_{s}-y_{s}^{*}\right\|_{\mathcal{B}_{h}}^{2} d s \\
& +\frac{4 M^{2} C_{1}^{2}}{\Gamma^{2}(\alpha)} \int_{0}^{t}(t-s)^{2(\alpha-1)} L_{\sigma}\left\|y_{s}-y_{s}^{*}\right\|_{\mathcal{B}_{h}}^{2} d s \\
& +\frac{12 M_{1} M_{2} M^{4} C_{1}^{4}}{\Gamma^{4}(\alpha)} \int_{0}^{t}(t-\eta)^{\alpha-1} d \eta \int_{0}^{t}(t-\eta)^{\alpha-1} \\
& \times\left[\int_{0}^{t}(t-s)^{(\alpha-1)} d s \int_{0}^{t}(t-s)^{(\alpha-1)} L_{f}\left\|y_{s}-y_{s}^{*}\right\|_{\mathcal{B}_{h}}^{2} d s\right] d \eta \\
& +\frac{12 M_{1} M_{2} M^{4} C_{1}^{4}}{\Gamma^{4}(\alpha)} \int_{0}^{t}(t-\eta)^{2(\alpha-1)}\left[\int_{0}^{t}(t-s)^{2(\alpha-1)} L_{\sigma}\left\|y_{s}-y_{s}^{*}\right\|_{\mathcal{B}_{h}}^{2} d s\right] d \eta \\
& +12 M_{1} M_{2} M^{2} C_{1}^{2} C_{2}^{2} L_{g}\left\|y-y^{*}\right\|_{\mathcal{B}_{h}}^{2} \\
& \leq\left(4 M^{2} C_{1}^{2} C_{2}^{2} L_{g} l+4 \frac{M^{2} C_{1}^{2}}{\Gamma^{2}(\alpha)} T^{2 \alpha}\left[\frac{L_{f} l}{\alpha^{2}}\left(1+\frac{3 M_{1} M_{2} M^{2} C_{1}^{2} T^{2 \alpha}}{\alpha^{2} \Gamma^{2}(\alpha)}\right)\right.\right. \\
& \left.\left.+\frac{L_{\sigma} l}{T(2 \alpha-1)}\left(1+\frac{3 M_{1} M_{2} M^{2} C_{1}^{2} T^{2 \alpha}}{T(2 \alpha-1) \alpha^{2} \Gamma^{2}(\alpha)}\right)\right]\right)\left\|y-y^{*}\right\|_{\mathcal{B}_{T}^{0}}^{2} \\
& =\Lambda\left\|y-y^{*}\right\|_{\mathcal{B}_{T}^{0}}^{2} \text {. }
\end{aligned}
$$


By the condition (3.3), we obtain that $\widehat{\Psi}_{2}^{*}$ is a contraction mapping. Hence, by Krasnoselskii's fixed point theorem we can conclude that the problem (4.1) has a mild solution on $(-\infty, T]$ and clearly, $x(T)=\left(\Psi^{*} x\right)(T)$, which implies that the system (4.1) is controllable on $(-\infty, T]$. This completes the proof of the theorem.

Example 4.4. Now, we present an example to illustrate Theorem 4.3. Consider the fractional partial stochastic differential equation in the following form

$$
\left\{\begin{array}{l}
D_{t}^{\alpha}\left[z(t, x)-z_{x x}(t, x)\right]=z_{x x}(t, x)+\mu(x, t)+F(t, z(t-R, x)) \\
+G(t, z(t-R, x)) \frac{d \omega(t)}{d t}, x \in=[0, \pi], R>0, t \neq t_{k} \\
I_{k}\left(z\left(t_{k}^{-}, x\right)\right)=z\left(t_{k}^{+}, x\right)-z\left(t_{k}^{-}, x\right), \quad k=1, \ldots, n \\
z(t, x)=\phi(t, x), \quad t \in(-\infty, 0], x \in[0, \pi] . \\
x(0, x)+\int_{0}^{\pi} H(x, y) z(t, y) d y=\phi(t, x), \quad t \in(-\infty, 0] .
\end{array}\right.
$$

where $\omega(t)$ is a standard cylindrical Wiener process in $\mathcal{H}$ defined on a stochastic space $\left(\Omega, \mathcal{F}, \mathbb{P},\left\{\mathcal{F}_{t}\right\}\right) ; D_{t}^{\alpha}$ is the Caputo fractional derivative of order $0<\alpha<1$; $0<t_{1}<t_{2}<\ldots<t_{n}<T$ are prefixed numbers. Let $h(t)=e^{2 t}, t<0$, then $l=\int_{-\infty}^{0} \frac{h(s)}{s} d s=\frac{1}{2}$ and define

$$
\|\phi\|_{\mathcal{B}_{h}}=\int_{-\infty}^{0} h(s) \sup _{s \leq \theta \leq 0}\left(E|\theta|^{2}\right)^{\frac{1}{2}} d s
$$

Let $\mathcal{K}=\mathcal{H}=L^{2}([0, \pi])$ with the norm $\|\cdot\|$. Define the operators $A: D(A) \subset \mathcal{H} \longrightarrow$ $\mathcal{H}, L: D(L) \subset \mathcal{H} \longrightarrow \mathcal{H}$, by $A z=-z^{\prime \prime}$ and $L z=z-z^{\prime \prime}$, where each domain $D(A)$ and $D(L)$ is given by $\left\{z \in \mathcal{H}, z, z^{\prime}\right.$ are absolutely continuous, $z^{\prime \prime} \in \mathcal{H}$ and $z(0)=$ $z(\pi)=0\}$.

Further, $A$ and $L$ can be $A z=\sum_{n=1}^{\infty} n^{2}\left(z, z_{n}\right) z_{n}, z \in D(A), L z=\sum_{n=1}^{\infty}(1+$ $\left.n^{2}\right)\left(z, z_{n}\right) z_{n}, z \in D(L)$, where $z_{n}(x)=\sqrt{\frac{2}{\pi}} \sin (n x), n=1,2, \ldots$, is the orthogonal set of vectors of $A$. Also, for $z \in \mathcal{H} L^{-1} z=\sum_{n=1}^{\infty} \frac{1}{\left(1+n^{2}\right)}\left(z, z_{n}\right) z_{n}, A L^{-1} z=$ $\sum_{n=1}^{\infty} \frac{n}{\left(1+n^{2}\right)}\left(z, z_{n}\right) z_{n}, T(t) z=\sum_{n=1}^{\infty} \exp \frac{n^{2} t}{\left(1+n^{2}\right)}\left(z, z_{n}\right) z_{n}$.

It is easy to see that $-A L^{-1}$ generates a uniformly continuous semigroup $T(t), t \geq 0$ and so $\max _{t \in[0, T]}\|T(t)\|$ is finite.

Hence for $(t, \phi) \in[0, T] \times \mathcal{B}_{h}$, where $\phi(\theta)(x)=\phi(\theta, x),(\theta, x) \in(-\infty, 0] \times[0, \pi]$. But $z(t)=z(t, \cdot)$, that is $z(t)(x)=z(t, x)$. Define $f:[0, T] \times \mathcal{B}_{h} \longrightarrow L^{2}([0, \pi])$, $\sigma:[0, T] \times \mathcal{B}_{h} \longrightarrow \mathcal{L}_{2}^{0}$. The bounded linear operator $B: \mathcal{U} \longrightarrow \mathcal{H}$ is defined by $B u(t)(x)=\mu(t, x), 0 \leq x \leq \pi, u \in \mathcal{U}$; as follows: 


$$
\begin{gathered}
g(z)(x)=\int_{0}^{\pi} H(x, y) z(t, y) d y, \\
f(t, \phi) x=F(t, x(\cdot)), \\
\sigma(t, \phi) x=G(t, x(\cdot)) .
\end{gathered}
$$

With the choice of $A, f, g$ and $\sigma$ can be rewritten as the abstract form of system (4.1). Under the appropriate conditions on the functions $f, \sigma, g$ and $I_{k}$ as those in (H1)-(H3) and (H4)-(H5), system (4.8) is controllable on $(-\infty, T]$.

\section{Acknowledgments}

The authors are very grateful to the referees and the Editor-in-Chief for their careful reading and helpful comments which helped to significantly improve the quality of the paper.

\section{References}

[1] H.M. Ahmed, Sobolev-Type Fractional Stochastic Integrodifferential Equations with Nonlocal Conditions in Hilbert Space, J. Theor. Probab. 30 (2017) 771-783.

[2] J. Bao, Z. Hou and C. Yuan, Stability in distribution of mild solutions to stochastic partial differential equations, Pro. American Math. Soc. 1386 (2010) 21692180 .

[3] J.H. Bao, Z.T. Hou, Existence of mild solutions to stochastic neutral partial functional differential equations with non-Lipschitz coefficients, Comput. Math. Appl. 59 (2010) 207-214.

[4] A. Benchaabane, R. Sakthivel, Sobolev-type fractional stochastic differential equations with non-Lipschitz coefficients, Journal of Computational and Applied Mathematics 312 (2017) 65-73.

[5] A. Boudaoui, A. Slama, Approximate controllability of nonlinear fractional impulsive stochastic differential equations with nonlocal conditions and infinite delay, Nonlinear Dynamics and Systems Theory 161 (2016) 35-48.

[6] J. Cao, Q. Yang, Z. Huang and Q. Liu, Asymptotically almost periodic solutions of stochastic functional differential equations, Appl. Math. Comput. 218 (2011) 1499-1511.

[7] J. Cao, Q. Yang and Z. Huang, On almost periodic mild solutions for stochastic functional differential equations, Nonlinear Anal. RWA 13 (2012) 275-286.

[8] M. Caputo, Elasticitáe Dissipazione, Zanichelli, Bologna, 1969. 
[9] Y.K. Chang, Z.H. Zhao, G.M. N'Guérékata and R. Ma, Stepanov-like almost automorphy for stochastic processes and applications to stochastic differential equations, Nonlinear Anal. RWA 12 (2011) 1130-1139.

[10] J. Dabas, A. Chauhan and M. Kumar, Existence of the mild solutions for impulsive fractional equations with infinite delay, Int. J. Differ. Equ., Volume 2011 (2011) Article ID 793023, 20 pages.

[11] A. Debbouche, J.J. Nieto, Sobolev type fractional abstract evolution equations with nonlocal conditions and optimal multi-controls, Applied Mathematics and Computation 245 (2014) 74-85.

[12] A. Debbouche, D.F.M. Torres, Sobolev type fractional dynamic equations and optimal multi-integral controls with fractional nonlocal conditions, Fractional Calculus and Applied Analysis 18 (2015) 95-121.

[13] M. Feckan, J. Wang and Y. Zhou, Controllability of fractional functional evolution equations of Sobolev type via characteristic solution operators, Journal of Optimization Theory and Applications 156 (2013) 79-95.

[14] I. Karatzas, S.E. Shreve, Brownian Motion and Stochastic Calculus, SpringerVerlag, Berlin 1991.

[15] M. Kerboua, A. Debbouche and D. Baleanu, Approximate controllability of Sobolev type fractional stochastic nonlocal nonlinear differential equations in Hilbert spaces, Electronic Journal of Qualitative Theory of Differential Equations 58 (2014) 1-16.

[16] M.A. Krasnosel'skii, Topological Methods in the Theory of Nonlinear Integral Equations, Pergamon Press, New York 1964.

[17] E. Lakhel, M.A. McKibben, Controllability of neutral stochastic integrodifferential evolution equations driven by a fractional Brownian motion. Afr. Mat. 7 (2010) 1-14.

[18] F. Li, J. Liang and H.K. Xu, Existence of mild solutions for fractional integrodifferential equations of Sobolev type with nonlocal conditions, Journal of Mathematical Analysis and Applications 391 (2012) 510-525.

[19] K. Li, J. Peng, Controllability of fractional neutral stochastic functional differential systems, Z. Angew. Math. Phys. 65 (2014) 941-959.

[20] N.I. Mahmudov, Existence and approximate controllability of Sobolev type fractional stochastic evolution equations, Bulletin of the Polish Academy of Sciences Technical Sciences 622 (2014) 205-215.

[21] X. Mao, Stochastic Differential Equations and Applications, Horwood, Chichester, UK 1997. 
[22] P. Revathi, R. Sakthivel and Y. Ren, Stochastic functional differential equations of Sobolev-type with infinite delay, Statistics and Probability Letters 109 (2016) 68-77.

[23] Y. Ren, D.D. Sun, Second-order neutral stochastic evolution equations with infinite delay under Carathéodory conditions, J. Optim. Theory Appl. 1473 (2010) 569-582.

[24] Y. Ren, Q. Zhou and L. Chen, Existence, uniqueness and stability of mild solutions for time-dependent stochastic evolution equations with Poisson jumps and infinite delay, J. Optim. Theory Appl. 149 (2011) 315-331.

[25] R. Sakthivel, P. Revathi and Y. Ren, Existence of solutions for nonlinear fractional stochastic differential equations, Nonlinear Analysis 81 (2013) 70-86.

[26] X.B. Shu, Y. Lai and Y. Chen, The existence of mild solutions for impulsive fractional partial differential equations, Nonlinear Anal. TMA 755 (2011) 20032011.

[27] A. Slama, A. Boudaoui, Approximate controllability of fractional impulsive neutral stochastic integro-differential equations with nonlocal conditions and infinite delay, Annals of Differential Equation 2 (2015) 127-139.

[28] F. Wei, K. Wang, The existence and uniqueness of the solution for stochastic functional differential equations with infinite delay, J. Math. Anal. Appl. 331 (2007) 516-531.

[29] Z. Yan, F. Lu, Existence results for a new class of fractional impulsive partial neutral stochastic integro-differential equations with infinte delay, Journal of Applied Analysis and Computation 53 August (2015) 329-346.

[30] Y. Zhou, F. Jiao, Existence of mild solutions for fractional neutral evolution equations, Comput. Math. Appl. 59 (2010) 1063-1077.

\title{
DOI: $10.7862 / \mathrm{rf} .2017 .3$
}

\author{
Ahmed Boudaoui \\ email: ahmedboudaoui@gmail.com \\ Department of Mathematics and Computer Sciences \\ University of Adrar \\ ALGERIA
}

Abdeldjalil Slama

email: slama_dj@yahoo.fr

Department of Mathematics and Computer Sciences

University of Adrar

ALGERIA

Received 18.04.2017 